\title{
Inversion Algorithms and PS Detection in SAR Tomography, Case Study of Bucharest City
}

\author{
Cosmin Dănişor, Student Member, IEEE, Gianfranco Fornaro, Senior Member, IEEE, and Mihai \\ Datcu, Fellow Member, IEEE
}

\begin{abstract}
Synthetic Aperture Radar (SAR) tomography can reconstruct the elevation profile of each pixel based on a set of co-registered complex images of a scene. Its main advantage over classical interferometric methods consists in the capability to improve the detection of single persistent scatterers as well as to enable the detection of multiple scatterers interfering within the same pixel. In this paper, three tomographic algorithms are compared and applied to a dataset of 32 images to generate the elevation map of dominant scatterers from a scene. Targets which present stable proprieties over time - Persistent Scatterers (PS) are then detected based on reflectivity functions reconstructed with Capon filtering.
\end{abstract}

Keywords - Beam-forming, Capon filtering, Least-Squares optimization, PS detection, SAR tomography

\section{INTRODUCTION}

$\mathrm{S}$ AR interferometry (InSAR) makes use of the phase difference between two complex valued images from different views of the same area. After separation of the topographic component from the interferometric phase, an accurate digital elevation model (DEM) of the scene can be generated.

The main limitations of InSAR are related to the fact that it does not possess any capabilities to separate the contribution of multiple targets from the same rangeazimuth resolution cell [1]. For example it fails in regions with steep slopes, like buildings' facades, the reconstructed DEM may be affected by layover [2]. In this case, the signal

Paper received May 3, 2016; revised May 27, 2016; accepted May 28, 2016. Date of publication July 20,2016. The associate editor coordinating the review of this manuscript and approving it for publication was Prof. Branimir Reljin.

This paper is a revised and expanded version of the paper presented at the 23rd Telecommunications Forum TELFOR 2015 [12].

The work has been funded by the Sectoral Operational Programme Human Resources Development 2007-2013 of the Ministry of European Funds through the Financial Agreement POSDRU/187/1.5/S/155536.

This work has been done in the frame of research project TOMOSAR PN-II-IDPCE-2011-3-1027.

Cosmin Dănişor is member of Department of Applied Electronics and Information Engineering at the University Politehnica of Bucharest (UPB), 1-3 Iuliu Maniu Avenue, 061071, Bucharest, Romania; (phone: 004021402 4623, e-mail: cosmin.danisor@yahoo.com).

Gianfranco Fornaro is Senior Researcher at the Institute of Electromagnetic Sensing of the Environment of National Research Council (CNR); 328 Via Diocleziano, 80124, Naples, Italy (phone: 0039 081762 0627, e-mail: fornaro.g@irea.cnr.it).

Mihai Datcu is Senior Scientist at Photogrammetry and Image Analysis Department of Remote Sensing and Technology Institute; German Aerospace Center (DLR), Oberpfaffenhofen, 82234 Wessling, Germany; (e-mail: mihai.datcu@dlr.de). from a single range-azimuth resolution cell contains the contributions of multiple echoes coming from different sources positioned in a different cell on ground. Residual components of interferometric phase, partly caused by temporal decorrelation and non-uniform propagation of waves through atmosphere, are also difficult to be estimated using only a pair of SAR images.

To overcome these limitations, SAR techniques using multiple acquisition were developed. Typically they involve variations of the view angle (multi-baseline) and are acquired over repeated passes (multi-temporal). Proper processing of stacks of SAR acquisitions allows the mitigation of noise and of error sources, as well as reduction of the phase ambiguity. They also form the basis of modern differential interferometry (D-InSAR) techniques, which measure and monitor the displacements of earth's surface over the time [1]. D-InSAR methods are commonly applied to study the effects of phenomena such as seismic and volcanic activities, landslides, glacier motions, mining, etc [3].

In order to be able to measure deformation rates, up to a fraction of the wavelength of radar signal, the differential component of interferometric phase (component related to scene's displacements) needs to be precisely estimated. For this, a technique which studies the phase variation of points which present stable electromagnetic proprieties over time was developed: PS-InSAR method [4]. Classical techniques employed for persistent scatterers' detection study the temporal variation of targets' amplitudes (indicated by an index of dispersion) and then the dispersion of the phase difference with respect to a model depending on the height and velocity of a scatterer.

\section{SAR TOMOGRAPHY}

SAR Tomography represents an extension of multitemporal interferometric techniques. It uses both the amplitude and phase of SAR acquisitions to reconstruct the distribution of scene's reflectivity function in elevation direction, in each resolution cell of the scene. The retrieved reflectivity function allows the improved detection of single persistent scatterers as well as the detection of multiple scatterers interfering within the same pixel. [5].

SAR images from the dataset need to be pre-processed before the implementation of tomographic methods. The first step consists of image co-registeration (preferably at subpixel level), so pixels located at the same range-azimuth position along the dataset will have the same component on the ground. Also, the residual component of the interferometric phase such as atmospheric disturbances 
must be estimated and subtracted, in order to minimize its possible distortions which can affect the reconstruction algorithms. Differential interferometry methods can be used for precise estimation of this component [4]

The basic system model of SAR tomography states that every pixel from the set of coregistered images represents the Fourier Transform of scene's reflectivity function, at a spatial frequency which depends on the perpendicular baseline of the image to which the pixel belongs [5]:

$$
g_{n k}=\int \gamma_{k}(s) e^{-j 2 \pi \xi_{n} s} d s, \quad \xi_{n}=-\frac{2 b_{p n}}{\lambda r}
$$

where $g_{n k}$ is the complex value of pixel $k$ from image $n ; \gamma_{k}(s)$ represents the variation in elevation direction of the reflectivity function of the $k^{\text {th }}$ pixel set, $\xi_{n}$ is the spatial frequency corresponding to the $n^{\text {th }}$ acquisition, $b_{p n}$ is the perpendicular baseline value between the master image and the $n^{\text {th }}$ image of the dataset, $\lambda$ is the radar frequency and $r$ denotes the distance between the sensor and scene's center at the moment of acquisition.

After coregistration, all pixels belonging to the same set $k$ will represent an identical portion of the scene. Reflectivity function's elevation resolution $\rho_{e l}$ depends on the span of perpendicular baseline values $b_{p r}[1]$ :

$$
\rho_{e l}=\frac{\lambda r}{2 b_{p r}}
$$

A normalized steering vector [5] $a$ can be defined at each point $s$ of the elevation, based on the values of spatial frequencies of the dataset:

$$
a(s)=\frac{1}{\sqrt{N}}\left(\begin{array}{llll}
e^{j 2 \pi \xi_{1} s} & e^{j 2 \pi \xi_{2} s} & \cdots & e^{j 2 \pi \xi_{N} s}
\end{array}\right)^{\mathrm{H}}
$$

where $\mathrm{H}$ is the Hermitian operator, and $N$ represents the number of images from dataset.

\section{OBJECTIVES}

The main objective of this work is to perform a comparative assessment of three tomographic algorithms: Beam-Forming, Least-Squares optimization and Capon filtering. These methods were applied to reconstruct the variation of reflectivity function in elevation direction starting from a dataset of complex SAR images. A preprocessing chain was also implemented to coregister the images and to subtract the residual interferometric phase of the test scene.

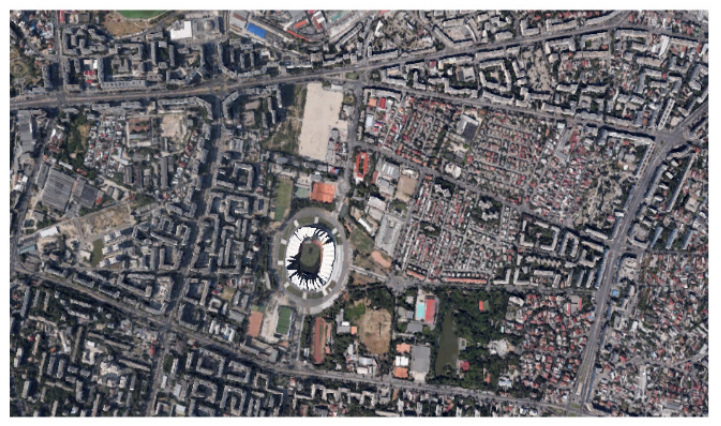

Fig. 1. Satellite view of test region.

The dataset is made of 32 complex images of Bucharest, Romania, acquired by German satellite TerraSAR-X in strip-map mode over an interval of one year and a half (July
2011 to December 2012). For the evaluation of the algorithms we employed a small area cut from the dataset, containing the National Arena and the surrounding neighborhood. An optical satellite view of the region is presented in Fig 1.

It can be noticed that the region consists mainly of buildings, so we expect a high density of PS.

Images acquired by TerraSAR-X satellite in strip-map mode have the azimuth resolution of $3.3 \mathrm{~m}$, and ground range resolution of $1.9 \mathrm{~m}$. The amplitude of the test scene from the master image is presented in Fig 2.

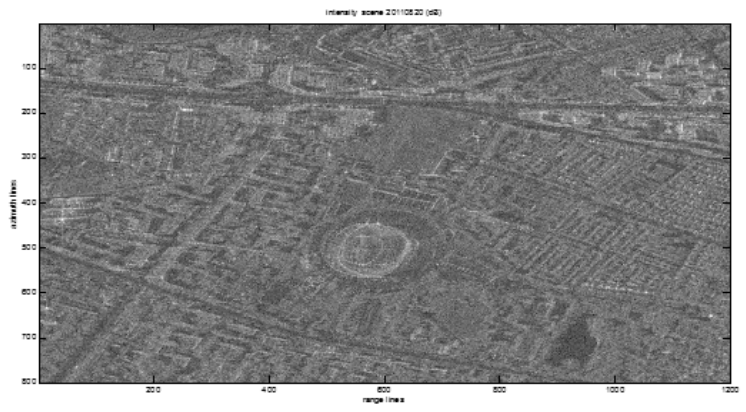

Fig. 2. Amplitude of the test scene from a TerrSAR-X image (acquired on 20.08.2011).

The reference image of the dataset (master image) was chosen so that the perpendicular baseline values between it (master) and the rest of the images (slave images) have a minimum dispersion. Under those conditions, the range of perpendicular baseline values equals $432 \mathrm{~m}$ - replacing TerraSAR-X acquisitions' specific parameters in equation (2) this leads to an elevation resolution cell of $23.3 \mathrm{~m}$.

In Fig 3 each image of the dataset is positioned in the plane defined by perpendicular baseline values and acquisition dates. It can be noticed that perpendicular baselines are irregularly distributed.

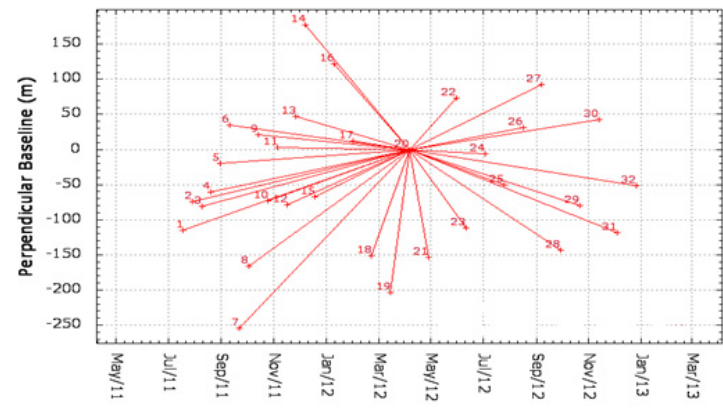

Fig. 3. Distribution of dataset's perpendicular baselines.

In the following sections, three tomographic algorithms (Beam-Forming, Least-Squares optimization and Capon filtering) will be implemented to reconstruct the reflectivity functions in each pixel from the selected region. The results of those three spectral estimation methods will be evaluated and compared by representing the elevation maps of the dominant scatterers. In each pixel, the height of the dominant scatterer is identified as the elevation point at which the reconstructed reflectivity function presents its maximum absolute value. Persistent scatterers of the scene will then be detected based on the reflectivity functions reconstructed with Capon filtering. 


\section{REFLECTIVITY FunCtions RECONSTRUCTION}

\section{A. Images pre-processing}

Coregistration of images from the dataset is necessary because each acquisition is conducted from a different position. To compensate the variation of acquisitions' geometries, the resulted offsets in range and azimuth between each slave image and master image need to be estimated. Offsets assessing is implemented for each master-slave pair by maximization of amplitude's correlation coefficient $R$ [6], which is calculated in various windows across the scene:

$$
R=\frac{\sum_{l \in w} I_{m}(l) I_{s}^{*}(l)}{\sqrt{\sum_{l \in w}\left|I_{m}(l)\right|^{2} \sum_{l \in w}\left|I_{s}(l)\right|^{2}}}
$$

where $I_{m}$ and $I_{s}$ represent the amplitudes of master and slave images, and $w$ denote the windows in which the coherence is calculated. After offset estimation, each slave image is resampled in order to overlap with the master image of the dataset.

An interferogram is obtained through performing a pixelby-pixel multiplication of the master image with the complex conjugate of a slave image. The interferometric phase $\Phi_{i t f}$ represents the difference between the two images phases and contains, besides the topographic component $\Phi_{\text {topo }}$, the contribution of Earth's surface curvature - $\Phi_{f l}$, a component denoting the deformations that occurred on the scene between acquisitions (both linear and non-linear) $\Phi_{d e f}$, effects of non-linear propagation of waves through atmosphere - $\Phi_{\text {atm }}$, and a noise component $\Phi_{\text {noise }}$ [4]:

$$
\Phi_{i t f}=\Phi_{f l}+\Phi_{\text {topo }}+\Phi_{\text {def }}+\Phi_{\text {atm }}+\Phi_{\text {noise }}
$$

In SAR tomography, the topographic component of interferometric phase is used as a starting point in combination with the absolute value of the images in the analyzed dataset. Therefore, this term must be separated from the interferometric phase. The component related to the curvature of Earth's surface can be estimated and subtracted knowing the trajectory of orbit's satellite during acquisitions [7] (interferogram flattening).

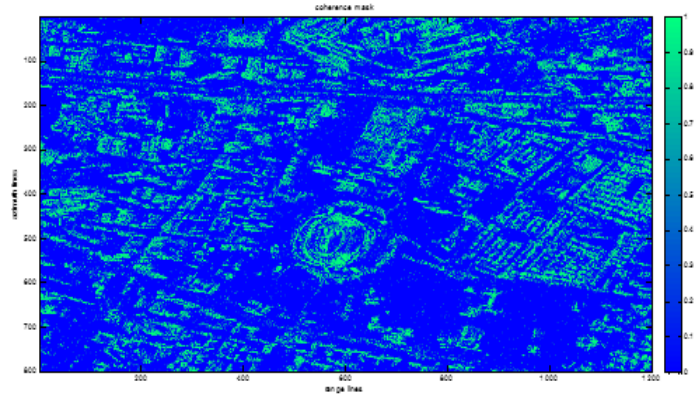

Fig. 4. Points with amplitude coherence above 0.5 .

Considering the relative small surface of the scene $(1.9 \times 3$ $\mathrm{km}^{2}$ ), the effects of atmospheric phase screen can be considered uniform over each image. In this case, the residual phase component is considered as being constant along each acquisition, and can be estimated as the pondered mean of flattened interferometric phase of points with a correlation index of amplitude above 0.5 . The resulting mask is presented in Fig 4.

Highlighted points represent a set of PS candidates identified based on amplitude's proprieties. As expected, points with a high amplitude coherence index are located mostly on roofs and facades of buildings.

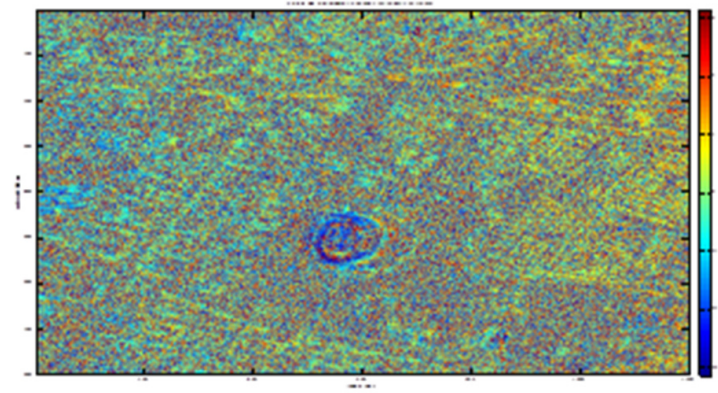

Fig. 5.Calibrated interferometric phase.

The flattened phase of each point from the mask is pondered by the associated correlation coefficient during the averaging process:

$$
e^{\mathrm{j} p h m}=\frac{1}{M} \sum_{l=1}^{M} R(l) e^{\mathrm{j} \cdot p h f(l)}
$$

where $M$ represents the number of points with amplitude's correlation coefficient $R$ above 0.5 and phf denotes the flattened interferometric phase. The estimated value of the residual phase, phm is subtracted from each point of the interferogram for phase calibration. Adjusted interferometric phase is exemplified in Fig 5.

Samples of reflectivity function's spectrum are located at spatial frequencies which depend on dataset's perpendicular baselines, as stated in equation (1). For each pixel, the number of available spectrum samples equals the number of dataset's images (in this case 32). Given the perpendicular baselines' set of values, samples are irregularly distributed, as exemplified in Fig 6.

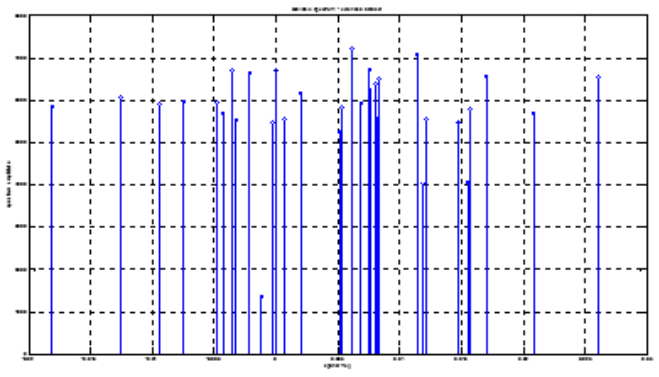

Fig. 6. Reflectivity function's available spectrum samples.

Variation of reflectivity function in elevation direction will be reconstructed for each resolution cell of the scene by application of spectral estimation methods. The reconstruction of the reflectivity function ranges in elevation - from -150 to $150 \mathrm{~m}$. This domain allows the detection of both dominant and secondary scatterers.

\section{B. Beam-Forming $(B F)$}

This method reconstructs the reflectivity function's variation by projecting a filter which doesn't distort the available components of its spectrum while attenuating as much as possible the components on other frequencies [2]. The first condition is equivalent with a unitary scalar product between filter's coefficients $h_{B F}$ and steering 
vector, at every point of elevation, as presented in (7). The second condition involves a choice of coefficients which minimize the output power of the filer, $P_{B F}(8)$ :

$$
\begin{gathered}
h_{B F}^{\mathrm{H}} a=1 \\
h_{B F}=\min _{h} P_{B F} \equiv \min _{h} h^{H} R_{g} h
\end{gathered}
$$

where $R_{g}$ represents the covariance matrix of the input data.

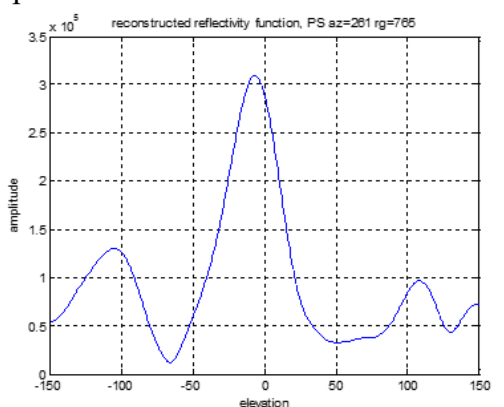

Fig. 7. Reflectivity function reconstructed with BF.

This method presumes that the input data is completely decorrelated $\left(R_{g}=I\right)$, in which case the filter coefficients equal the elements of the normalized steering vector from (3). In Fig 7 is presented the variation of filter's output power, having the spectrum samples exemplified in Fig 6 as input. A 3 by 3 multi-look (ML) window was used for estimation of covariance matrix. This reflectivity function contains three distinct local peaks, so the corresponding resolution cell presents three distinct scatterers in the reconstruction interval of the elevation.

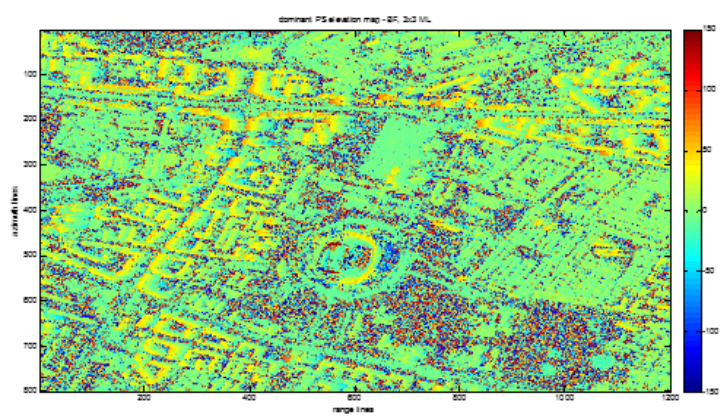

Fig. 8. Elevation map generated with Beam-Forming method, using $3 \times 3 \mathrm{ML}$.

The elevation map generated through Beam-Forming filtering is presented in Fig 8. The shape of the objects (buildings and stadium) is observable in regions with a high index of amplitude's coherence.

\section{Least-Squares optimization}

The relation between the reflectivity function and its spectrum can be written in matrix form:

$$
g=R \gamma
$$

where $g$ represents the vector of available spectrum samples, $R$ denotes the exponents' matrix, and $\gamma$-vector of reflectivity function's samples. The number of reflectivity function values we want to retrieve is much higher than the number of available spectrum samples, therefore the system from (9) is underdetermined. In order to find its optimal solution, we consider the cost function which represents the norm of error's samples vector:

$$
J(\gamma)=\|g-R \gamma\|^{2}
$$

By minimizing this cost function (cancelling its first derivative) we find the optimal solution of the system in least-squares (LS) sense [8]:

$$
\gamma_{L S}=\left(R^{T} R\right)^{-1} R^{T} g
$$

The product between exponents' matrix transpose and the matrix itself is rank-deficient, therefore it isn't invertible. To be able to calculate the optimal solution, a regularization parameter needs to be added to the cost function defined in (10). Using the singular values decomposition (SVD) $\left(u_{i}, \sigma_{i}, v_{i}\right)$ of the exponents' matrix, the optimal LS solution becomes [8]:

$$
\gamma_{L S}=\sum_{i=1}^{N} \frac{\sigma_{i}}{\sigma_{i}^{2}+\alpha^{2}}\left(u_{i}^{H} g\right) v_{i}
$$

where $\alpha$ represents the regularization parameter.

The solutions of this method are strongly dependent on the value of regularization parameter. If this parameter has a small value, equation (12) is equivalent with a direct SVD inversion of regularization matrix, which doesn't prevent noise propagation, as can be seen in elevation map presented in Fig 9:

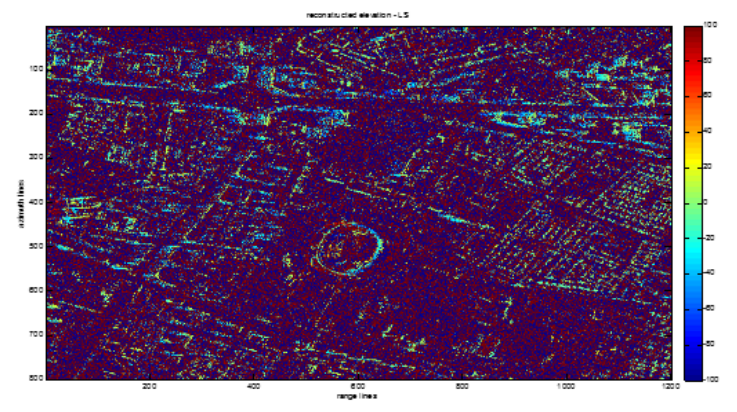

Fig. 9. Elevation map generated with LS optimization, using a low regularization parameter, no ML.

As the value of the regularization parameter increases, the optimal LS solution becomes increasingly closer to the solution of Beam-Forming filter. Therefore, the reconstructed elevation maps begin to resemble the one generated by Beam-Forming method.

A usual choice for the regularization parameter is the estimated noise level value, in which case the LS optimization becomes equivalent to SVD-Wiener noise filtering [9]. For noise level approximation, reflectivity function's available spectrum samples $g$ are projected in exponent's matrix singular vectors space. Noise level $\varepsilon$ is estimated as [10]:

$$
\varepsilon=\sqrt{\frac{N}{n_{\varepsilon}} \sum_{i=N-n_{\varepsilon}+1}^{N} u_{i}^{T} g}
$$

Only last $n_{\varepsilon}$ projections are taken into consideration for noise evaluation. Parameter $n_{\varepsilon}$ is determined by analyzing the singular values spectrum (SVS) of exponent's matrix $R$, whose samples $S_{i}$ are computed as:

$$
S_{i}=\sigma_{i} / \sigma_{1}, \quad i=1: N
$$

and represented in Fig 10: 


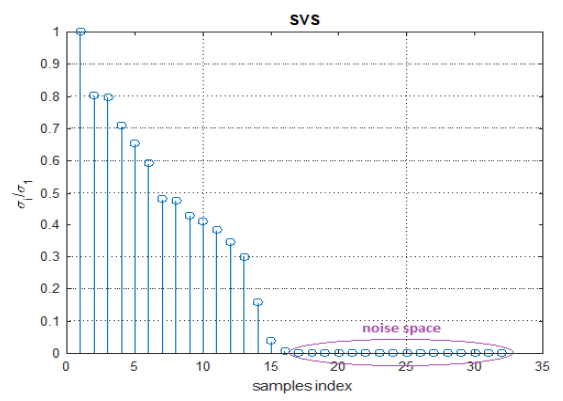

Fig. 10. Singular values spectrum of exponents' matrix.

Because of the nature of singular values $\sigma_{i}$, SVS samples are decreasing with each incrimination of index $i$. SVS values close to zero are deemed as belonging to noise space [9], so only the afferent projections will be considered for estimation of noise level $\varepsilon$. In our case, noise threshold for SVS was chosen as $10^{-3}$, therefore only last three spectrum values formed the noise space $\left(n_{\varepsilon}=16\right)$, as shown in Fig 10 .

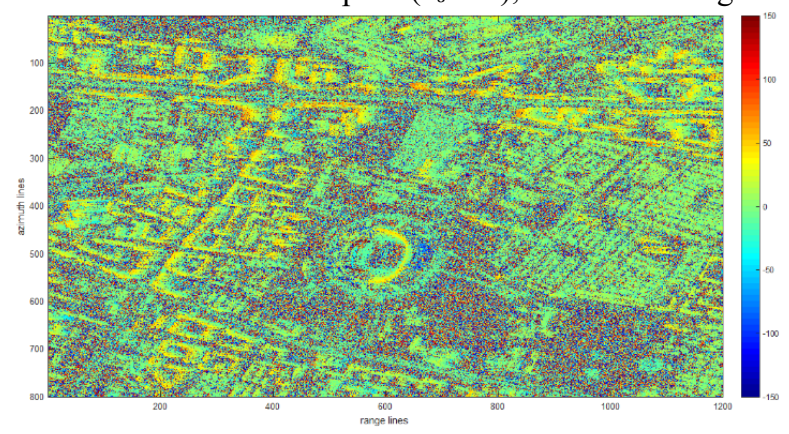

Fig. 11. Elevation map generated with SVD-Wiener filtering, no ML.

Elevation map of dominant scatterers generated through LS optimization, when regularization parameter equals the estimated noise level, is presented in Fig 11. This is the only implemented reconstruction algorithm in which no multilooking is applied.

\section{Capon filter}

The main limitation of Beam-Forming method is related to the fact that the input data was assumed to be totally decorrelated, but in SAR tomography this assumption isn't always valid. The Capon filter is designed to overcome this limitation, while respecting the same two principles stated in equations (7) and (8). In this case, filter's coefficients have the following form [2]:

$$
h_{c}(s)=\frac{R_{g}^{-1} a(s)}{a^{H}(s) R_{g}^{-1} a(s)}
$$

and the output power of the filter becomes [2]:

$$
P_{C}(s)=\frac{1}{a(s)^{H} R_{g}^{-1} a(s)}
$$

The covariance matrix of the input data can be estimated as:

$$
R_{g}=\frac{1}{N} g \cdot g^{H}
$$

therefore when using a 3 by 3 multi-look window, the matrix is rank-deficient and needs to be regularized in order to admit an inverse. A usual regularizing method is the diagonal loading:

$$
R_{g r}=R_{g}+\lambda I
$$

where regularization parameter $\lambda$ can be chosen as the mean variance of elements form input data vector:

$$
\lambda=\frac{1}{N} \operatorname{trace}\left(R_{g}\right)
$$

Elevation map reconstructed by Capon filtering is presented in Fig 12:

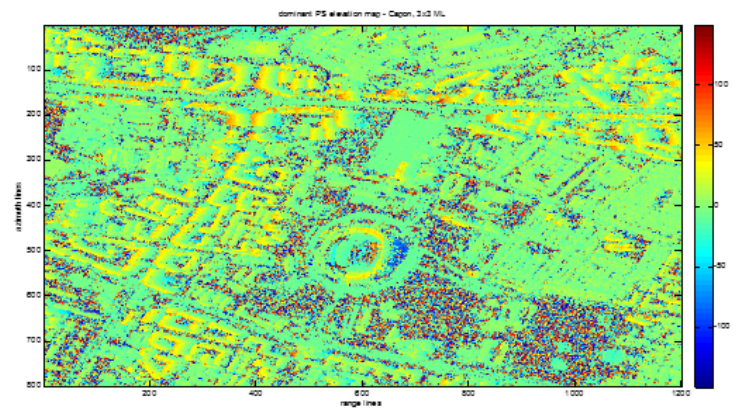

Fig. 12. Elevation map generated by Capon filtering with 3x3 ML window.

On close comparison with the results of Beam-Forming method, it can be noticed that noise level is reduced after Capon filtering. Both methods were implemented using the same multi-look window dimensions.

\section{E. Spectral estimation algorithms overview}

The main difference between the results of the three tomographic algorithms presented in this section consists in the noise level affecting the elevation maps. Capon filtering has slightly better performances than Beam-Forming method, while the results of LS optimization are strongly dependent on a regularization parameter value.

In areas with a high correlation index of amplitude, the elevation values of dominant scatterers reconstructed by those three methods are similar, close to reference values indicated by Google Earth.
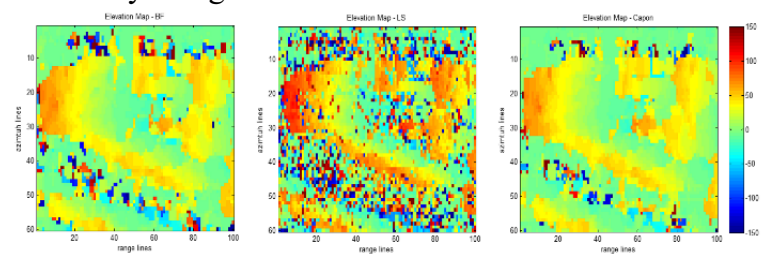

Fig. 13. Reconstructed elevation for a stable structure: BF (left), LS optimization (center) and Capon (right).

For the region of two buildings presented in Fig 13, the absolute mean difference between the heights reconstructed by the three methods has the following values: $7.64 \mathrm{~m} \mathrm{(BF}$ and Capon), $19.48 \mathrm{~m}$ (BF and LS) and $21.93 \mathrm{~m}$ (Capon and LS). However, those values are affected by the presence of noise, so in order to compare the elevation values reconstructed across the entire scene, stable targets need to be separated from the ones affected by noise. This operation can be realized by implementation of persistent scatterers detection algorithms, which are presented in the following section. 


\section{Persistent Scatterers Detection}

As previously mentioned, Persistent Scatterers denote stable targets, which present low temporal decorrelation. These points are identified by studying the variation of targets proprieties over an extended temporal interval, using a dataset of multiple SAR acquisitions. PS are intensely used in differential interferometry because of their potential to generate highly accurate measurements [4]. The main steps of PS-InSAR technique include the estimation of atmospheric phase screen (interferometric component related to waves' nonuniform propagation through atmosphere), generation of precise digital elevation models [3], and finally measurements of scene's deformation, which can be done with millimeter accuracy. [7].

Persistent scatterers can also be identified by an analysis of reflectivity functions reconstructed with SAR tomography. The position of dominant scatteres was identified in each resolution cell by identification of the position of reflectivity's maximum absolute value. In the case of Beam-Forming and Capon filtering methods, the nature of those scatterers can be estimated by calculation of the correlation index between the input data and filter's coefficients. By imposing a threshold to the correlation index, coherent targets can be separated from the ones affected by noise. Correlation index between Capon's filter coefficients and input data is:

$$
c i_{C}=\frac{\left|h_{C}\left(s_{\max }\right)^{H} g\right|}{\left\|h_{C}\left(s_{\max }\right)\right\|\|g\|}
$$

where $\|\cdot\|$ denotes the L2-norm of the vector and $s_{\max }$ represents the identified height of dominant scatterers. For better separation of stable targets from the ones affected by noise, the squared form of this correlation index can be used:

$$
c i_{C}^{2}=\frac{\left|h_{C}\left(s_{\max }\right)^{H} R_{g} h_{C}\left(s_{\max }\right)\right|}{\left\|h_{C}\left(s_{\max }\right)\right\|^{2} \operatorname{trace}\left(R_{g}\right)}
$$

Targets with a squared form of correlation index above 0.5 were classified as persistent scatterers. Spatial distribution of identified PS is presented in Fig 14

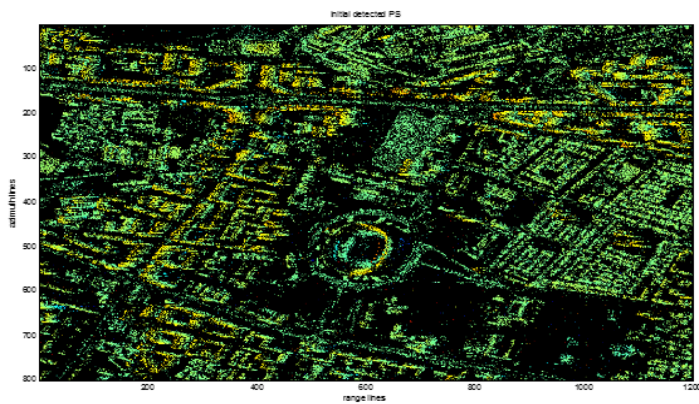

Fig. 14. Identified Persistent Scatterers.

As expected, persistent scatterers are mostly detected on scene's stable structures, like building's facades and roofs. Areas of the scene affected by temporal decorrelation, like vegetation regions, do not contain targets with stable electromagnetic proprieties over time.

\section{CONCLUSIONS}

Three tomographic algorithms (Beam Forming, LeastSquares optimization and Capon filtering) were implemented on a set of 32 SAR images to reconstruct the variation of scene's reflectivity functions in elevation direction. Results of those three spectral estimation methods were evaluated by representation of identified dominant scatterers' elevation maps, the immediate conclusion being that the main difference between those techniques is related to noise filtering capability.

To be able to compare the algorithm's results across the entire scene, targets with low temporal decorrelation were separated from the ones affected by noise. An algorithm for persistent scatterers detection was implemented based on reflectivity functions reconstructed by Capon filtering.

Mean absolute differences between elevation maps of identified persistent scatterers, generated with the three mentioned spectral estimation methods are: $4.15 \mathrm{~m}$ (BF and Capon), $13.73 \mathrm{~m}$ (BF and LS) and 15.61 m (Capon and LS).

Future methods address alternative PS detection algorithms, using Generalized Likelihood Ratio Test [11] and Magnitude Squared Coherence index. Techniques for dominant PS's contribution extraction will be applied to detect the presence of secondary PSs across the scene's resolution cells. Non-Linear Least Squares algorithm will be applied in points where secondary scatterers are detected, to simultaneously refine the elevations of both primary and secondary targets.

\section{REFERENCES}

[1] G. Fornaro, A. Pauciullo, D. Reale and S. Verde, "Multilook SAR Tomography for 3-D Reconstruction and Monitoring of Single Structures Applied to COSMO-SKYMED Data," in IEEE J. Sel. Topics Appl. Earth Observ. in Remote Sens, vol. 7, no. 7, pp. 2776-2785, July 2014.

[2] F. Gini, F. Lombardini and M. Montanari, "Layover solution in multibaseline SAR interferometry," in IEEE Trans. Aerosp. Electron. Syst, vol. 38, no. 4, pp. 1344-1356, Oct 2002.

[3] O. Mora, R. Arbiol, V. Pala, A. Adell and M. Torre, "Generation of Accurate DEMs Using DInSAR Methodology (TopoDInSAR)," in IEEE Geosci. Remote Sens. Lett., vol. 3, no. 4, pp. 551-554, Oct. 2006.

[4] A. Ferretti, C. Prati and F. Rocca, "Permanent scatterers in SAR interferometry," in IEEE Trans. Geosci. Remote Sens., vol. 39, no. 1, pp. 8-20, Jan 2001

[5] G. Fornaro, "Tomographic SAR", NATO SET-191 Lecture Series, Bucharest 10-11 November 2014

[6] M. S. Seymour and I. G. Cumming, "Maximum likelihood estimation for SAR interferometry," IEEE Int. Geosci. Remote Sens. Symp., 1994., Pasadena, CA, 1994, pp. 2272-2275, vol. 4.

[7] A. Ferretti, C. Prati and F. Rocca, "Nonlinear subsidence rate estimation using permanent scatterers in differential SAR interferometry," in IEEE Trans. Geosci. Remote Sens., vol. 38, no. 5, pp. 2202-2212, Sep 2000.

[8] G. H. Golub and C. Reinsch, "Singular value decomposition and least squares solutions," Numerische Mathematik, vol. 14, no. 5, pp. 403-420, 1970 .

[9] X. X. Zhu and R. Bamler, "Sparse tomographic SAR reconstruction from mixed TerraSAR-X/TanDEM-X data stacks," 2012 IEEE Int. Geosci. Remote Sens. Symp., Munich, 2012, pp. 7468-7471.

[10] X. X. Zhu and R. Bamler, "Very High Resolution Spaceborne SAR Tomography in Urban Environment," in IEEE Trans. Geosci. Remote Sens., vol. 48, no. 12, pp. 4296-4308, Dec. 2010.

[11] A. Budillon and G. Schirinzi, "GLRT Based on Support Estimation for Multiple Scatterers Detection in SAR Tomography," in IEEE J. Sel. Topics Appl. Earth Observ. in Remote Sens, vol. 9, no. 3, pp. 1086-1094, March 2016.

[12] C. Dănişor, G. Fornaro and M. Datcu, "Comparative study of SAR tomographic reconstruction algorithms," Telecommunications Forum Telfor (TELFOR), 2015 23rd, Belgrade, 2015, pp. 437-440. 